\title{
Étude de l'évolution des bateaux et convois soumis aux courants traversiers et au vent, à l'entrée du canal de liaison Fos-Rhône
}

\section{Study of the behaviour of boats and units under cross-current and wind action at the entrance to the Fos-Rhone canal link}

\author{
J. Megard et P. F. Demenet \\ SOGREAH
}

L'étude sur modèle réduit de la jonction du Rhône et du canal de navigation projeté entre le Rhône et la zone industrielle et portuaire de Fos a été réalisée par Sogreah pour le Port Autonome de Marseille (cf. plan de situation, fig. 1).

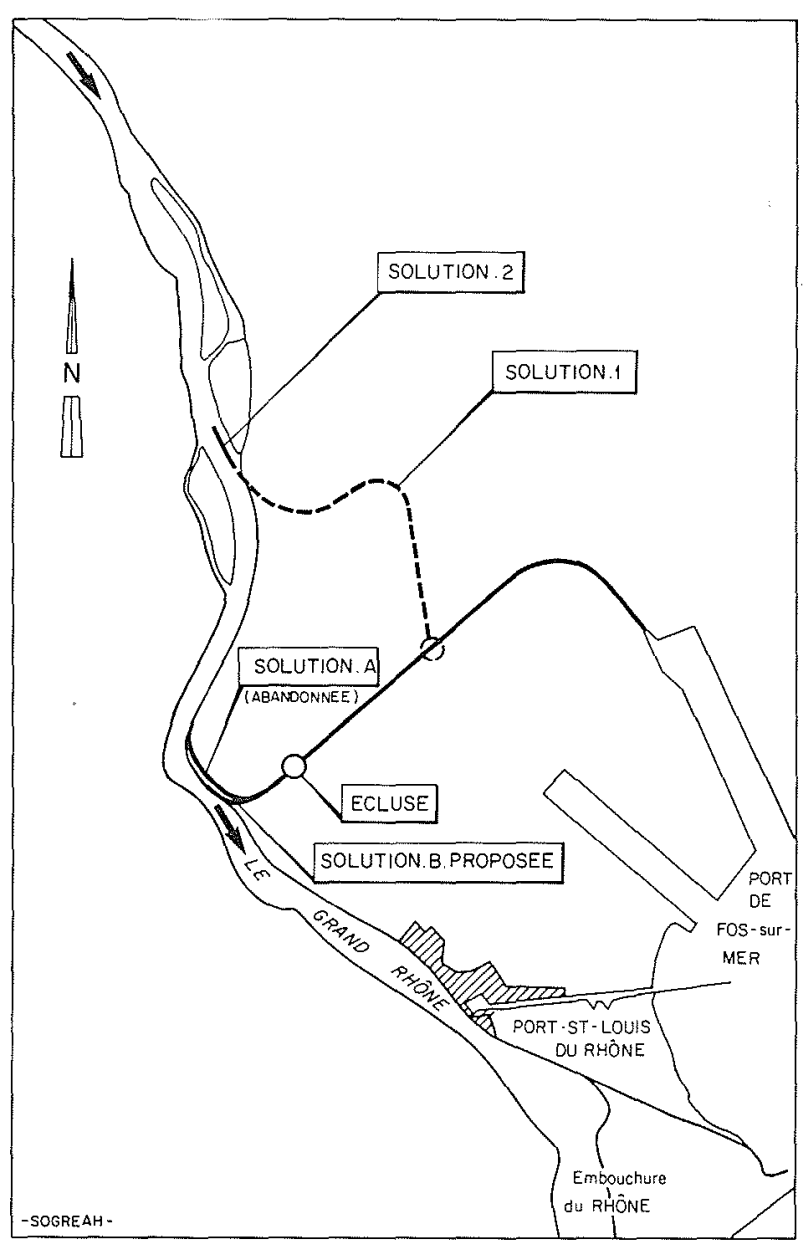

Figure 1 - Plan de situation

Les objectifs recherchés étaient les suivants :

- maintien de profondeurs suffisantes pour les convois poussés;

- obtention de conditions acceptables pour la mancuvre des convois poussés dans le système de courants obtenus.

Le programme d'étude comprenait deux étapes :

- la première étape était l'étude sur modèle du maintien des profondeurs par différents ouvrages (épis, seuils...) avec observation du champ des courants; - la seconde étape était le contrôle de la navigabilité à l'aide d'épures du mouvement des convois, dans les champs de courant observés.

Nous traiterons dans une première partie des problèmes spécifiques au modèle réduit physique : description du modèle et des méthodologies de l'observation des courants et de la sédimentation, bref historique des différentes variantes d'entrée étudiées sur le modèle.

Une deuxième partie concernera la méthodologie de l'étude de navigabilité et donnera des exemples d'application

Modèle réduit physique

\section{Description du modèle}

Le modèle représentait le lit du Rhône entre les PK 309,5 et 318,3 . La photographie en donne une vue générale.

Les échelles étaient les suivantes:

Echelle verticale

Echelle horizontale

Echelle des vitesses

Echelle des débits

Echelle des temps hydrauliques

Echelle des temps de sédimentation

$$
\begin{aligned}
\lambda & =1 / 100 \\
\mu & =1 / 150 \\
\lambda^{1 / 2} & =1 / 10 \\
\lambda^{3 / 2} \mu & =1 / 150000 \\
\mu / \lambda^{1 / 2} & =1 / 15 \\
1 / 300 & \text { à } 1 / 750
\end{aligned}
$$

LA HOUILLE BLANCHE/N² 2/3-1981 


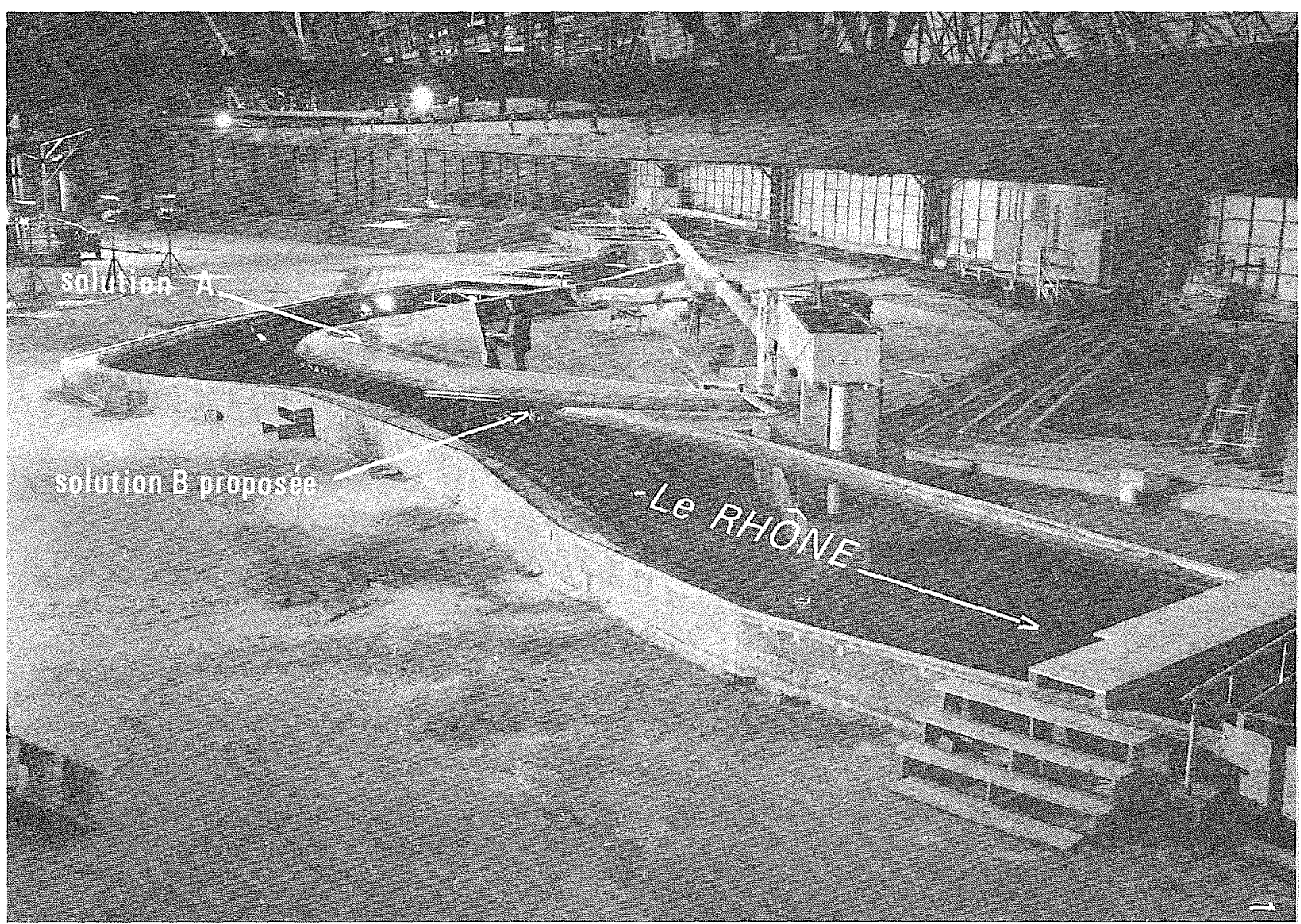

Vue générale đu modèle.

Le lit était constitué de sciure calibrée D60-80, de diamètre moyen $0,2 \mathrm{~mm}$. L'alimentation du modèle en eau et en sciure était faite en circuit fermé. L'équipement du modèle permettait de régler et de mesurer les niveaux et les débits.

L'échelle des temps de sédimentation a été obtenue à partir de raisonnements semi-théoriques. Les volumes de matériaux trouvés sur le modèle sont cohérents avec ceux que drague la CNR dans ses garages d'écluses.

\section{Méthodologie de l'observation des courants}

Les champs des courants ont été relevés sur le modèle pour les débits de 1400 et de $4000 \mathrm{~m}^{3} / \mathrm{s}$ dans le grand Rhône. Le débit de $1400 \mathrm{~m}^{3} / \mathrm{s}$ correspond sensiblement au débit médian du Rhône. Le débit de $4000 \mathrm{~m}^{3} / \mathrm{s}$ n'est dépassé que deux jours par an en moyenne. Il correspond à $4500 \mathrm{~m}^{3} / \mathrm{s}$ dans le Rhône total et il est à la limite supérieure des eaux navigables.

Le relevé des champs de courant a été obtenu par chronophotographie.

Toutes les photos de courants ont été faites en présence de fonds modelés suivant les profils en travers du lit réel ou du canal; ceci permettait d'éliminer les influences parasites qui auraient pu provenir des dunes de sciure du modèle ou des différences locales du modèle des fonds.

\section{Méthodologie de l'observation de la sédimentation}

Le relevé des fonds obtenus sur le modèle a été fait par photographie de courbes de niveau mises en place au contour de plans d'eau successifs.

L'état du lit photographié a été obtenu par deux méthodes.

a) la première méthode avait pour objectif de rechercher l'équilibre limite du lit et des dépôts qui se réalisaient sur le modèle. Elle consistait à faire fonctionner le modèle à $3000 \mathrm{~m}^{3} / \mathrm{s}$ pendant 36 heures environ. Elle résultait des essais de réglage, selon lesquels on avait estimé que ce débit était celui qui représentait le mieux sur le modèle l'ensemble des débits variables du Rhône du point de vue du modelé des fonds. La durée de 36 heures était suffisante pour que les fonds du modèle atteignent pratiquement leur modelé d'équilibre. A la fin, on faisait passer en outre une crue de 40 minutes, qui atteignait un maximum de $8000 \mathrm{~m}^{3} / \mathrm{s}$ et qui avait l'avantage de compléter les dépôts par les suspensions et surtout de lisser les dunes de sciure et de permettre ainsi une meilleure définition des fonds.

Cette méthode était adéquate pour caractériser l'équilibre du lit. Elle était employée pour comparer le modèle et la réalité et pour comparer les différentes solutions d'entrée.

b) la deuxième méthode a été employée pour la mise au point de la solution retenue (solution $\mathrm{B}$ ). Elle répondait 


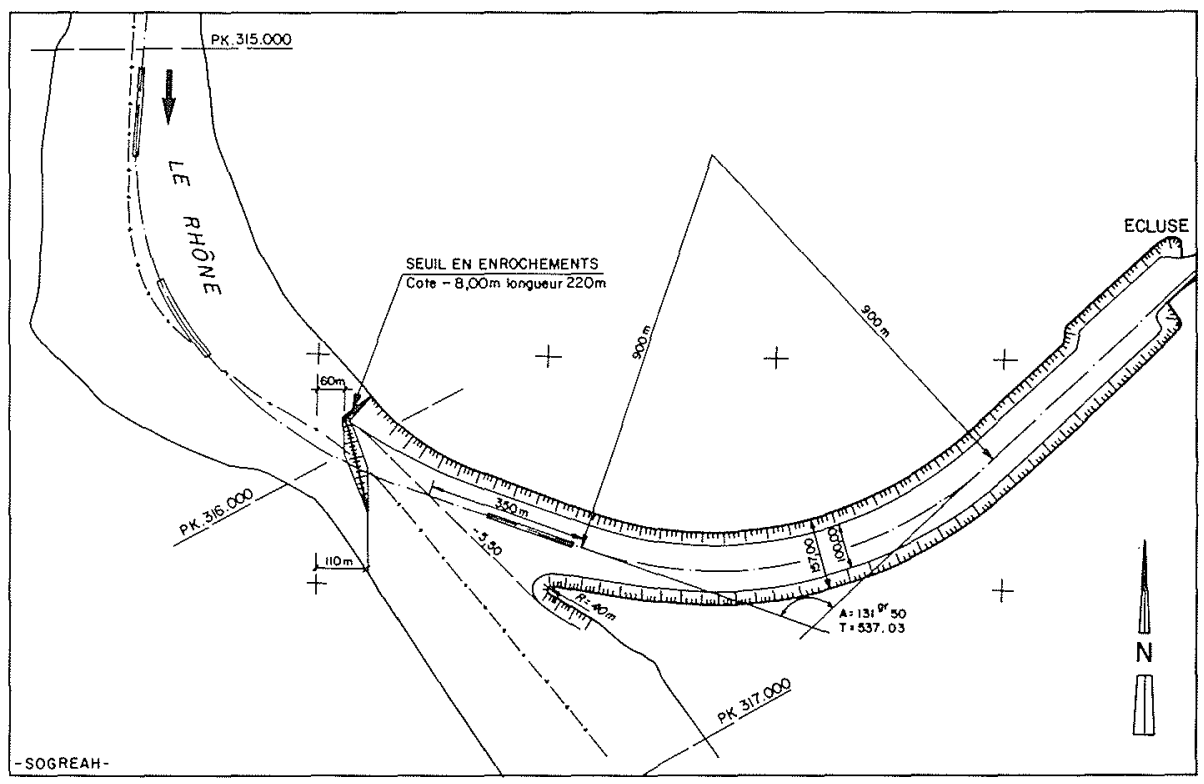

Figure 2 - Formes d'entrée mises au point pour le site $B$

au désir du Port Autonome de mieux identifier l'importance relative des dépôts qui devaient se former aux débits ordinaires et en crue. Son objectif était essentiellement de repérer le rythme des dépôts à éliminer par des dragages et non la limite qu'ils atteindraient si on ne les enlevait pas.

\section{Historique des variantes d'entrée étudiées sur le modèle}

Des contraintes essentiellement d'ordre foncier ont obligé le Port Autonome de Marseille à abandonner les solutions 1 et 2 (cf. Fig. 1).

Deux nouveaux sites d'entrée ont été définis (sites A et B, cf. Fig. 1). L'étude sur modèle des courants, affouillements et dépôts à l'entrée, ainsi que l'étude sur plan de la navigabilité ont montré que les deux sites d'entrée étaient valables.

Le site $A$ présentait de légers avantages techniques, mais le site $\mathrm{B}$ a été retenu en raison de son moindre coût.

$\mathrm{La}$ collaboration entre PAM et Sogreah a alors abouti aux deux solutions représentées sur la figure 2 :

- une solution de base sans seuil ;

- une variante, qui consiste à ajouter à la solution de base un seuil en enrochements noyé dans le Rhône (cote -8$)$. Il réduit le dépôt de sédiments à l'entrée et améliore légèrement la navigation.

\section{Bases de l'étude de la navigabilité}

\section{Généralités}

L'étude de manœuvrabilité d'un convoi sur le site et dans les conditions envisagées aurait pu être effectuée sur le modèle utilisé pour l'étude sédimentologique avec une maquette propulsée et téléguidée. Ce procédé se heurtait aux difficultés suivantes : complication et coût de la maquette à réaliser (notamment en ce qui concerne l'appareil propulsif); distorsion du modèle du Rhône; difficultés d'une représentation correcte du pilotage par un pilote situé à terre, hors du pousseur ; représentation du vent.

D'autre part, un modèle mathématique intégral de la mancuvre du convoi aurait été probablement aussi coûteux, en prix et delais, que le précédent.

On a finalement eu recours à une étude sur plan semiempirique, basée d'une part sur l'expérience de gens ayant la pratique de manœuvres effectuées dans des conditions analogues (notamment les pilotes des convois), d'autre part sur l'exécution de trajectographies de la mancuvre d'entrée, à partir de considérations cinématiques et dynamiques.

Les données existantes concernant la manœuvrabilité et l'expérience des pilotes ont été recueillies auprès des compagnies Sogestran et Sanara, notamment au cours de deux missions:

- descente du Rhône d'Avignon à Port-Saint-Louis avec un convoi lège Simbeu +2 barges, escales à Arles (Société Sogestran);

- Visite du remorqueur Emperi en cale de réparation à Marseille (Société Sanara).

\section{Caractéristiques des convois}

Deux types de convois poussés circulent actuellement sur le Rhône :

- les convois armés par la Sogestran, société de gestion et de transports fluviaux (siège : Le Havre) dont la flotte comporte actuellement 2 pousseurs, le "Simbeu" de $3000 \mathrm{CV}$ et le "Brau" de $2000 \mathrm{CV}$.

Chaque pousseur propulse 2 barges de dimensions principales : longueur $76,50 \mathrm{~m} \times$ largeur $11,40 \mathrm{~m}$ - tirant d'eau maximal $3,20 \mathrm{~m}$ - port en lourd 2200 à $2300 \mathrm{t}$ à ce tirant d'eau ;

- les convois armés par les sociétés Sanara, Citerna et Rhodania, comportent un pousseur "Empéri" de $2880 \mathrm{CV}$ ou "Reiaume" et des barges pour marchan- 
dises générales et hydrocarbures lourds, groupées par 2 et de caractéristiques principales :

longueur $80 \mathrm{~m} \times$ largeur $11,30 \mathrm{~m}$ - tirant d'eau maxi $4,25 \mathrm{~m}$ correspondant à un port en lourd de $2975 \mathrm{t}$.

L'apparition de nouveaux types de convois dans le futur ne peut modifier sensiblement ces données, les dimensions de ceux-ci étant limitées par le gabarit des écluses du Rhône, fixé par la CNR à $200 \mathrm{~m} \times 12 \mathrm{~m}$.

Le seul développement envisageable consisterait dans l'emploi éventuel sur le bas Rhône de convois à 4 barges, avec nécessité de découpler pour le franchissement des écluses.

La manœuvrabilité de ces convois diffère sensiblement d'un convoi à l'autre en fonction des équipements de propulsion et de gouverne dont dispose chacun, soit : - pour les pousseurs Sogestran

- 3 hélices orientables,

- plans de dérive escamotables à l'avant.

- pour les pousseurs Sanara :

- 2 hélices en tuyères,

- pousseur transversal à l'avant.

On retiendra seulement ici les caractéristiques principales utilisées dans l'étude :

- convoi du type Sanara, à pousseur "Empéri", qui présente les dimensions maximales et des qualités manœuvrières un peu moins poussées que celles du convoi Sogestran :

$\begin{array}{ll}\text { Longueur } & 190 \mathrm{~m} \\ \text { Largeur } & 11,4 \mathrm{~m} \\ \text { Tirant d'eau lège } & 0,65 \mathrm{~m} \\ \text { Surface latérale offerte au vent } & 800 \mathrm{~m}^{2} \\ \text { Hauteur moyenne sur l'eau } & 4,20 \mathrm{~m}\end{array}$

- situation lège. Il est d'expérience que les difficultés dues à l'accroissement des effets du vent l'emportent sur celles dues à l'inertie en situation pleine charge.

-.Vitesse au cours de la manœuvre d'entrée : $10 \mathrm{~km} / \mathrm{h}$

- distance d'arrêt : $450 \mathrm{~m}$

- Giration : diamètre : $300 \mathrm{~m}$

vitesse de giration : 0,5 à $1 \%$

- Temps de montée en allure :

- Stoppé à lent $(600 \mathrm{tr} / \mathrm{mn})=7 \mathrm{~s}$

- lent - route

$$
=15 \mathrm{~s}
$$

\section{Conditions d'environnement retenues pour l'étude}

\section{Définition du vent}

On a utilisé les observations des vents à l'aéroport d'Istres-le-Tube au cours des périodes 1949-1958 et 1962-1970, ainsi que les observations faites à la Fossette par le Port Autonome de Marseille au cours des années 1974 à 1978 .

L'analyse des données a conduit à adopter comme vent type pour l'étude, un vent de $15 \mathrm{~m} / \mathrm{s}$ venant de l'azimut $345^{\circ}$, qui n'est dépassé que $0,8 \%$ du temps en moyenne.

Les épures de navigation ont été réalisées aussi pour le cas d'un vent nul.

\section{Conditions de courant}

Elles ont été déterminées sur le modèle réduit à partir des chronophotos de visuels flottant en surface. Les écoulements ont été enregistrés pour le débit de $4000 \mathrm{~m}^{3} / \mathrm{s}$ (dépassé 2 jours par an en moyenne) et pour le débit médian de $1400 \mathrm{~m}^{3} / \mathrm{s}$.

\section{Méthodologie des épures de navigation}

L'établissement des trajectoires que suivront les convois au cours de la mancuvre d'entrée dans le canal comprend :

- le calcul de la dérive due au vent, en prenant en compte les efforts sur le gouvernail ;

- l'exécution pas à pas du graphique des vitesses sur le fond, compte tenu des courants.

\section{Calcul de la dérive due au vent}

Sur le navire en route, doué d'une certaine vitesse en avant, la dérive se traduit par un angle d'incidence $\beta$ entre le cap du navire et sa route sur l'eau. L'action de l'effort transversal engendré par le vent, $F y$, est équilibrée par l'apparition d'une portance hydrodynamique $P$ et d'un effort transversal sur le système de gouverne Fyg.

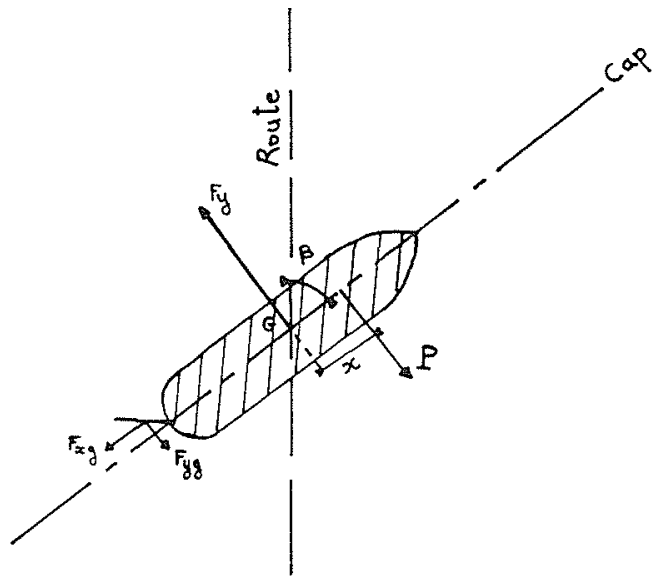

Le point d'application de la portance se trouve à une distance $x$ du centre géométrique, si bien que l'on a la relation suivante entre $F y g$ et $p$ :

$$
P . x=F y g . \frac{L}{2}
$$

$L$ étant la longueur du convoi.

Pour un convoi lège et un vent transversal, le point d'application $F y$ est sensiblement au milieu de la longueur.

L'équilibre des forces donne :

$$
F y g+P=F y
$$

d'où, en tenant compte de la relation (1)

$$
F y=P(1+2 x / L)
$$

Les expressions des deux forces $F y$ et $P$ sont les suivantes:

$$
\begin{aligned}
& F y=\frac{\rho a}{2} C y A l(W r)^{2} \\
& P=\frac{\rho e}{2} C^{\prime} y A s(V n)^{2}
\end{aligned}
$$


avec :

$\rho a$ : masse spécifique de l'air

pe : masse spécifique de l'eau

$W r$ : vitesse du vent relatif

$V n$ : vitesse du navire sur l'eau

As : projection de la surface de carène immergée sur le plan axial

$A l$ : projection de la surface émergente sur le plan axial

$C y$ : coefficient aérodynamique

$C^{\prime} y$ : coefficient de portance

Les valeurs de $C y$ dépendent de l'angle d'incidence $\alpha$ du vent relatif par rapport à l'axe du navire. Les valeurs adoptées sont données par la figure 3 .

Le coefficient de portance $C^{\prime} y$ est égal à $K c . \beta$ et dépend de la profondeur relative (profondeur/tirant d'eau). On a adopté pour la constante $K c$ la valeur de 0,013 pour l'ensemble des calculs effectués.

L'angle de dérive $\beta$ a donc pour valeur :

$$
\beta=\frac{\rho a}{\rho e} \frac{C y}{K c} \frac{A l}{A s}\left(\frac{W r}{V n}\right)^{2} \frac{1}{(1+2 x / L)}
$$

Lors du calcul des trajectoires, $W r$ et $\alpha$ sont déterminés par composition graphique des vitesses. On en déduit, grâce à la courbe de la figure 3 , une valeur de $C y$. Le rapport $x / L$ est obtenu, à l'aide de l'abaque de la figure 4 , en fonction de l'angle de dérive $\beta$. Comme dans la relation (6) apparaît implicitement dans le nombre de droite l'angle de dérive $\beta$, on est obligé de procéder par itérations pour calculer cet angle. En pratique, il y a convergence au bout de la deuxième itération.

L'effort transversal sur le système de gouverne Fyg peut être ensuite aisément calculé, en fonction de $P$ et de $x / L$.

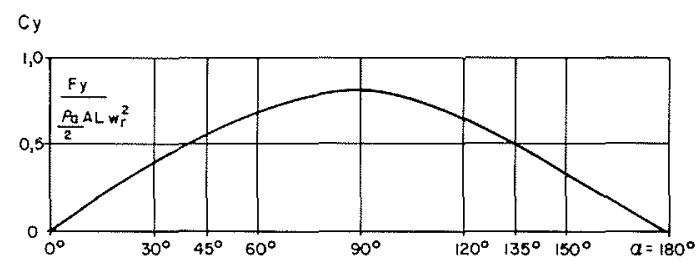

Figure 3 - Efforts dus au vent - Coefficient aérodynamique

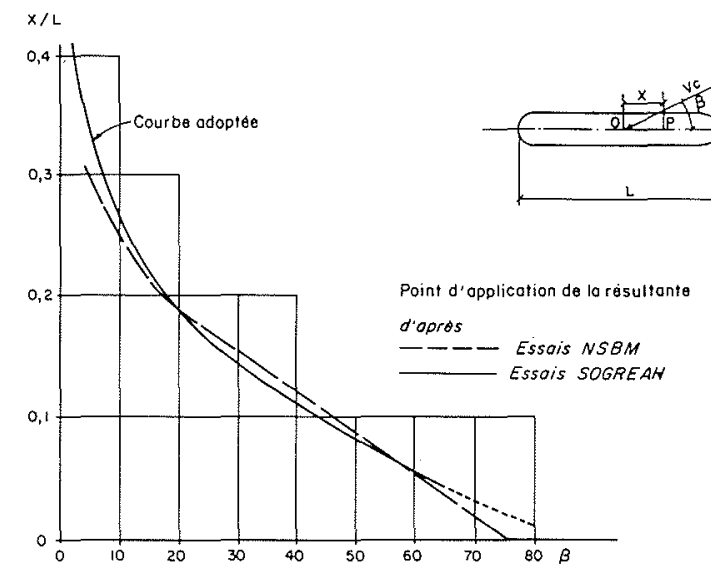

Figure 4 - Efforts dus au courant - Point d'application de la résultante.

\section{Calcul de la dérive due au courant}

La vitesse du navire et la vitesse du courant sont composées graphiquement. Il en résulte un angle de dérive $\beta_{c}$ et une vitesse sur le fond.

\section{Dérive totale}

La dérive totale est obtenue en additonnant les deux dérives $\beta$ et $\beta_{c}$.

\section{Franchissement d'une frontière du courant}

Le franchissement d'une frontière du courant a été abordé de manière très simplifiée par l'estimation des moments subis par la carène dans la zone de courants et la zone calme. On a pu montrer que la variation maximale de cap résultante pour un convoi lège animé d'une vitesse sur l'eau de $10 \mathrm{~km} / \mathrm{h}$ et une vitesse de courant de $1 \mathrm{~m} / \mathrm{s}$ était de l'ordre de 1 à $2^{\circ}$ après $15 \mathrm{sec}$. de pénétration dans la zone calme, du moins dans le domaine de variation des paramètres exploré lors des études de trajectographie.

Il s'agit là d'ordre de grandeur, ne tenant pas compte des réactions du pilote. Celui-ci devra contrer cette tendance en mettant de la barre à droite, manœuvre, de plus, nécessaire pour rapprocher le cap de l'axe de route du fait de la diminution de la dérive. Il en résulte un déportement sur babord avant de rejoindre l'axe de route.

\section{Exemples d'application}

Il s'agit des épures obtenues :

- avec la solution $B$. de base, un débit du Rhône de $1400 \mathrm{~m}^{3} / \mathrm{s}$ et un vent nul (Fig. 5 ),

- avec la solution $B$ de base, un débit du Rhône de $1400 \mathrm{~m}^{3} / \mathrm{s}$ et un vent de $15 \mathrm{~m} / \mathrm{s}$ (Fig. 6),

- avec la solution $B$ avec seuil, un débit du Rhône de $1400 \mathrm{~m}^{3} / \mathrm{s}$ et un vent de $15 \mathrm{~m} / \mathrm{s}$ (Fig. 7),

- avec la solution $B$ avec seuil, un débit du Rhône de $4000 \mathrm{~m}^{3} / \mathrm{s}$ et un vent de $15 \mathrm{~m} / \mathrm{s}$ (Fig. 8).

Sur les épures des figures 5 et 6 , les conditions sur les champs de courants rencontrés le long de la trajectoire sont les suivantes :

- vitesse maximale : $1,15 \mathrm{~m} / \mathrm{s}$,

- vitesse moyenne de l'ordre de $0,85 \mathrm{~m} / \mathrm{s}$,

- annulation de la vitesse assez brusque à l'entrée du canal.

Le convoi commence par longer les hauts fonds sur la rive droite, à une distance d'à peu près $125 \mathrm{~m}$ de celleci, vitesse sur l'eau ralentie à $10 \mathrm{~km} / \mathrm{h}$; sa vitesse sur le fond est alors de $13 \mathrm{~km} / \mathrm{h}$.

En suivant cette route, le convoi se présente à peu près dans l'axe de l'entrée du canal.

Le convoi traverse ensuite le lit, pour gagner l'entrée du chenal sur la rive gauche. Cette mancuvre a pour conséquence de lui donner une certaine obliquité par rapport au courant. La dérive atteint alors $11^{\circ} 5$ avec vent et $5^{\circ}$ sans vent. 

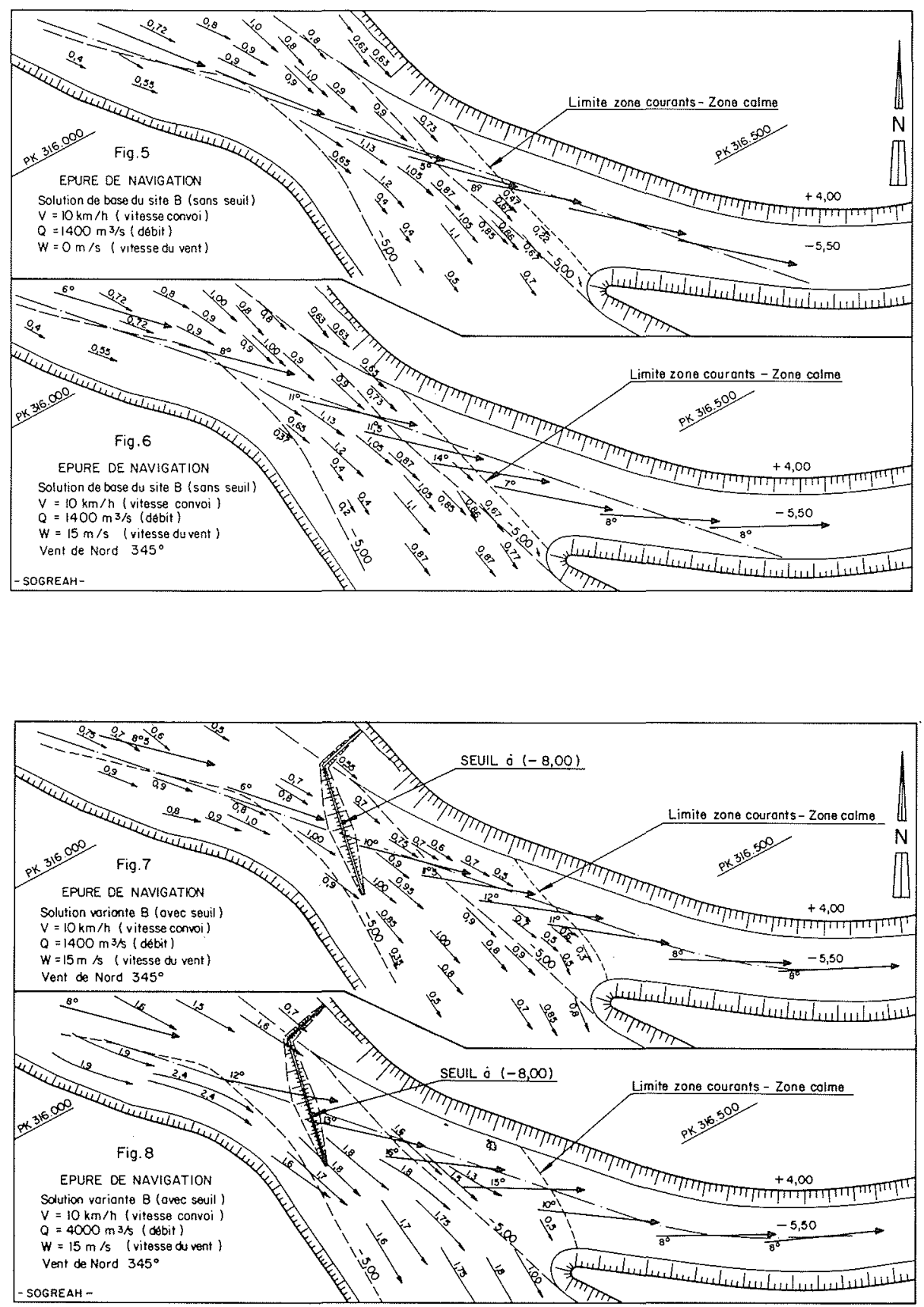

A partir du $\mathrm{km} 316,300$, le navire amorce une abattée sur babord de l'ordre d'une quinzaine de degrés et franchit la frontière entre la zone des courants et la zone calme. L'incidence du vent s'accroît alors légèrement et les angles de dérive avec et sans vent s'élèvent respectivement à $14^{\circ}$ et $8^{\circ}$.

$\mathrm{Au}$ fur et à mesure de l'entrée dans la zone calme, la vitesse sur le fond diminue jusqu'à $10 \mathrm{~km} / \mathrm{h}$ et la dérive décroît progressivement du fait de la disparition des courants, elle atteint $8^{\circ}$ dans le canal en présence de vent et s'annule complètement dans le cas contraire.

Pour la solution avec seuil (Fig. 7), la manœuvre d'approche est sensiblement identique à la précédente. On peut cependant constater que l'on obtient avec cette solution des angles de dérive moins importants. l'angle de dérive maximal avec vent est en effet de $12^{\circ}$ au lieu de $14^{\circ}$ précédemment.

Les courants sont déviés par le seuil et pénètrent plus profondément à l'intérieur du canal. Cette déviation est favorable à la navigation car, pendant toute la traversée du lit, l'obliquité du convoi par rapport au courant est moins grande. De plus, on constate une légère diminution des intensités des vitesses dans cette même région.

Les conditions sur les champs de courant rencontrés le long de la trajectoire sont les suivantes :

- vitesse maximale : $0,9 \mathrm{~m} / \mathrm{s}$;

- vitesse moyenne de l'ordre de : $0,75 \mathrm{~m} / \mathrm{s}$. 
Un autre effet bénéfique apporté par le seuil est une transition plus progressive entre la zone des courants et la zone calme.

Pour un débit de $4000 \mathrm{~m}^{3} / \mathrm{s}$ (Fig. 8), l'évolution générale de la manœuvre d'approche reste similaire. L'angle de dérive maximal avec vent atteint maintenant $16^{\circ}$, avec comme conditions sur les champs de courant :

- vitesse maximale : $2,20 \mathrm{~m} / \mathrm{s}$;

- vitesse moyenne : $1,70 \mathrm{~m} / \mathrm{s}$

\section{Conclusion}

L'étude de la jonction du Rhône et du canal de navigation projeté entre le Rhône et la zone industrielle et portuaire de Fos représente la combinaison et la synthèse d'une étude sur modèle réduit, de recueils d'observations en nature, d'enquêtes auprès de navigants et d'une étude sur plan semi-empirique, basée sur les données recueillies et sur des considération théoriques. 